\title{
Recognition of Completely Mixed Games
}

\author{
A. J. Goldman
}

(November 14, 1962)

\begin{abstract}
A matrix game is called completely mixed if no optimal strategy has a zero component. J. von Neumann's necessary and sufficient "separated diagonals" condition for a 2-by-2 game to be completely mixed was extended by Bohnenblust, Karlin, and Shapley to a sufficient condition for a general matrix game to be completely mixed. The present paper gives still weaker sufficient conditions, thus facilitating recognition of a wider class of completely mixed games as such. Special stress is put on the possibility of using row and column permutations to transform a given matrix into one obeying the conditions.
\end{abstract}

\section{Introduction}

Let $A=\left(a_{i j}\right)$ be an $m$-by- $n$ matrix with $i=0,1$, ..., $m-1$ and $j=0,1, \ldots, n-1$. We regard $A$ as the payoff table of a finite zero-sum two-person game; if Player I chooses his $i$ th course of action and Player II chooses his $j$ th course of action, then the outcome of the game is such that the rules prescribe a payment of $a_{i j}$ units to Player I by Player II. (If $a_{i j}<0$, the "physical" payment would go in the opposite direction.)

An $m$-component row vector $X=\left(x_{0}, \ldots, x_{m-1}\right)$ such that

$$
\sum_{i=0}^{m-1} x_{i}=1, \quad \text { all } x_{i} \geq 0
$$

can be interpreted as a mixed strategy (i.e., a probabilistic mixture of courses of action) for Player I, with $x_{i}$ representing the relative frequency or probability with which he employs his ith course of action. Similarly, an $n$-component column vector $Y$ with nonnegative components $y_{j}$ summing to unity can be interpreted as a mixed strategy for Player II. The bilinear form $X A Y$ then represents the expected value of the payoff to Player I by Player II, if they select the respective mixed strategies $X$ and $Y$.

A triple $\left(v, X^{*}, Y^{*}\right)$, where $v$ is a number and $X^{*}$ and $Y^{*}$ are mixed strategies for Players $I$ and II respectively, is called a solution of the game if

$$
X A Y^{*} \leq v \leq X^{*} A Y
$$

holds for all mixed strategies $X$ and $Y$. The celebrated minimax theorem of J. von Neumann asserts the existence of at least one solution. The number $v$ is called the value of the game; though equal to $X^{*} A Y^{*}$, it can be shown to be independent of the particular solution. A mixed strategy is called optimal if it appears in at least one solution, and it is known that a mixed strategy $X^{*}$ is optimal if and only if

$$
\sum_{i=0}^{m-1} x_{i}^{*} a_{i j} \geq v \quad \text { for } j=0,1, \ldots, n-1,
$$

while a mixed strategy $Y^{*}$ is optimal if and only if

$$
\sum_{j=0}^{n-1} a_{i j} y_{j}^{*} \leq v \quad \text { for } i=0,1, \ldots, m-1 .
$$

We will make use of the easily proved fact that if strict inequality holds in (1) for some particular $j$ and any optimal $X^{*}$, then $y_{j}^{*}=0$ for every optimal $Y^{*}$, while if strict inequality holds in (2) for some particular $i$ and any optimal $Y^{*}$, then $x_{i}^{*}=0$ for every optimal $X^{*}$.

The game is called completely mixed if every optimal strategy, for each player, has all its components strictly positive. Intuitively this means that depriving a player of one of his courses of action would really damage him versus a rational opponent. It is known ${ }^{1,2}$ that a completely mixed game must have a square matrix, and so $m=n$ will be assumed in what follows.

For $0 \leq k \leq n-1$ let $D_{k}$ denote the $k$-th diagonal of $A$, the set of entries $a_{i j}$ with $j-i=k(\bmod n)$. A "classical" result of von Neumann ${ }^{3}$ asserts that a 2-by-2 game is completely mixed if and only if its diagonals are separated, i.e., if there are disjoint intervals $I_{0}$ and $I_{1}$ with $D_{0} \subset I_{0}$ and $D_{1} \subset I_{1}$. A generalization was given by Bohnenblust, Karlin, and Shapley ${ }^{4}$ who showed that an $n$-by- $n$ game is completely mixed if its diagonals are both separated and ordered, i.e., if there are disjoint intervals in the order

${ }^{1}$ I. Kaplansky, A contribution to von Neumann's theory of games, Annals of Math. 46 (1945)

\& D. Gale and S. Sherman, Solutions of finite two-person games, Paper 4 of Princeton Annals of Math. Study No. 24 (1950), Contributions to the Theory of Games. See p. 48 .

$3 \mathrm{O}$. Morgenstern and J. von Neumann, Theory of Games and Economic Behavior, Princeton University Press (1947). See p. 173.

4 H. F. Bohnenblust, S. Karlin, and L. S. Shapley, Solutions of discrete twoperson games, Paper 5 of Princeton Annals of Math 'Study No. 24 (1950), Conperson games, Paper 


$$
I_{0}<I_{1}<\ldots<I_{n-1}
$$

such that $D_{k} \subset I_{k}$ for $0 \leq k \leq n-1$. They point out, however, that this sufficient condition is not necessary for complete mixture even when $n=3$.

It can be shown (op. cit. in footnote 4) that a game with payoff matrix $\boldsymbol{A}$ is completely mixed if and only if the same is true of all games whose matrices are obtainable from $A$ by a sequence of operations each of one of the following types:

(i) Permutation of rows.

(ii) Permutation of columns.

(iii) Matrix transposition.

(iv) Matrix negation.

The diagonals-separated-and-ordered condition (briefly, the "BKS condition") for complete mixture may apply to one of these transforms of $A$ but not to $A$ itself, so that a priori the criterion can only be used "fully" by testing the diagonals of each transform. It is fairly obvious that actual generation of all the transforms of $A$ can be replaced by a more efficient procedure, and theorem 1 of section 2 can be viewed as the rather straightforward justification of one such procedure, or alternatively as a determination of just how far the range of applicability of the BKS theorem is extended by the operations (i) through (iv).

Theorem 2 in section 3 gives a new sufficient condition for complete mixture. Though strictly weaker than the BKS condition (entries of $A$ in the same diagonal are not all lumped together), it is in the same general spirit, permitting a wider class of completely mixed games to be recognized as such "by inspection" if this term is generously interpreted. The ideas of section 2 are carried over to this new context, and theorem 3 deals with the possibility of transforming a given matrix into one obeying the sufficient condition of theorem 2.

Theorems 1,2, and 3 are the main results of the paper. Three related topics are treated in section 4 . First, a sufficient condition for complete mixture apparently still weaker than that of theorem 2 is given (theorem 4), but is shown (in theorem 5) to be equivalent when both conditions are aided by the transformations (i) through (iv). Second, it is shown by example that our results are not implied by a second sufficient condition for complete mixture due to Bohnenblust, Karlin, and Shapley. Third, it is noted that our criteria still fail to identify all $3 \times 3$ completely mixed games, in part because they apply only when each row and each column of the payoff matrix consists of distinct entries. Two results are given which require only "one-way" distinctness (theorem 6 for columns, theorem 7 for rows), but they do not resolve the case $n=3$.

\section{Testing the Applicability of the BKS Condition}

First a simplification will be made. Suppose a sequence of operations, of the types (i) through (iv) listed in the introduction, leads from a matrix $A$ to a matrix $A^{\prime}$ and contains exactly $t$ matrix transpositions and exactly $N$ matrix negations. Both transposition and negation are involutions, i.e., $\left(B^{T}\right)^{T}=B$ and $-(-B)=B$. Also, a row (column) permutation followed by a transposition is equivalent to the same permutation applied to the columns (rows) preceded by a transposition, while any row or column permutation commutes with negation. From these remarks it follows that $A^{\prime}$ can be obtained by applying an appropriate sequence, consisting of row and column permutations only, to

$\begin{aligned} A & \text { for } t \text { even, } N \text { even, } \\ -A & \text { for } t \text { even, } N \text { odd, } \\ A^{T} & \text { for } t \text { odd, } N \text { even, } \\ -A^{T} & \text { for } t \text { odd, } N \text { odd. }\end{aligned}$

We shall therefore delete transposition and negation from the repertoire of allowable operations; a transform of $A$ now will mean a matrix obtainable from $A$ by a sequence of row and/or column permutations. If $C$ denotes a necessary and sufficient condition that at least one transform of $A$ has some property, then the statement

$$
\text { " } A \text { or }(-A) \text { or } A^{T} \text { or }\left(-A^{T}\right) \text { satisfies } C "
$$

is a necessary and sufficient condition that at least one matrix obtainable from $A$ by all the operations (i) through (iv) of the introduction has the property.

Next some terminology and notation will be introduced. A pair of matrix entries ${ }^{5}$ is called collinear if the entries lie in the same row or the same column. A set of $n$ entries (in an $n$-by- $n$ matrix $A=\left(a_{i j}\right)$ ) will be called a chord if it contains no collinear pair; the chords of $A$ are precisely the sets of the form

$$
S(A, \pi)=\left\{a_{i, \pi(i)}: i=0,1, . . ., n-1\right\},
$$

where $\pi$ is a permutation of $\{0,1, . ., n-1\}$, determined uniquely by the chord. For example, the diagonals of $A$ are the sets

$$
D_{k}(A)=S\left(A, \delta_{k}\right) \quad k=0,1, \ldots, n-1
$$

associated with the "diagonal permutations" $\delta_{k}$ defined by

$$
\delta_{k}(i)=i+k \quad(\bmod n) .
$$

Suppose $A^{\prime}=\left(a_{i j}^{\prime}\right)$ is obtained from $A=\left(a_{i j}\right)$ by a row permutation $\sigma$ and a column permutation $\tau$, so that $a_{i j}=a_{\sigma(i), \tau(j)}^{\prime}$. Setting $t=\sigma(i)$ leads to

$$
a_{i, \pi(i)}=a_{t, \tau \pi \sigma^{-1}(t)}^{\prime} \in S\left(A^{\prime}, \tau \pi \sigma^{-1}\right),
$$

and it is easily shown by such reasoning that

$$
S(A, \pi)=S\left(A^{\prime}, \tau \pi \sigma^{-1}\right)
$$

5 To avoid cumbersome notation, we occasionally slur the distinction between a matrix entry $a_{\text {ij }}$ and its position $(i, j)$; this should cause no confusion. 
for any permutation $\pi$. If $A^{\prime}$ is obtained from $A$ by a sequence consisting of row permutations $\sigma_{1}, \sigma_{2}, \ldots$, $\sigma_{p}$ and column permutations $\tau_{1}, \tau_{2}, \ldots ., \tau_{q}$, each listed in their order of appearance in the sequence, then eq (4) still holds with the definitions ${ }^{6}$

$$
\sigma=\sigma_{1} \sigma_{2} . . \sigma_{p}, \quad \tau=\tau_{1} \tau_{2} \ldots \tau_{q} .
$$

This fact will be used in the following proof.

Theorem 1: Some transform of A has separated and ordered diagonals, if and only if

(i) the sets $\mathrm{S}_{\mathrm{k}}=\left\{\mathrm{a}_{\mathrm{r}}: \mathrm{kn} \leq \mathrm{r}<(\mathrm{k}+1) \mathrm{n}\right\}$ determined by an enumeration $\left\{\mathrm{a}_{\mathrm{r}}: 0 \leq \mathrm{r}<\mathrm{n}^{2}\right\}$ of the entries of $\mathrm{A}$ in nondecreasing order are independent of the particular enumeration, ${ }^{7}$

(ii) each $\mathrm{S}_{\mathrm{k}}$ is a chord, and thus determines a permutation $\pi_{\mathrm{k}}$ such that $\mathrm{S}_{\mathrm{k}}=\mathrm{S}\left(\mathrm{A}, \pi_{\mathrm{k}}\right)$, and

(iii) $\pi_{\mathrm{k}} \pi_{0}^{-1}=\left(\pi_{1} \pi_{0}^{-1}\right)^{\mathrm{k}}$ for $\mathrm{k}=0,1, \ldots, \mathrm{n}-1$.

The necessity of the conditions will be proved first. Suppose $A^{\prime}$ is a transform of $A$ which has separated and ordered diagonals. The set of entries of $A^{\prime}$ (multiplicities included) is the same as for $A$, so that

$$
S_{k}=\nu_{k}\left(A^{\prime}\right) \quad \text { for } k=0,1, \ldots, n-1 .
$$

This shows that the sets $S_{k}$ are unambiguously defined. Since row and column permutations preserve collinearity of pairs and therefore map only chords into chords, and since $D_{k}\left(A^{\prime}\right)$ is a chord of $A^{\prime}$, its "pretransform" $S_{k}$ must be a chord of $A$. Thus only the necessity of (iii) remains to be verified.

Since $A^{\prime}$ is a transform of $A$, there are permutations $\sigma$ and $\tau$ such that eq (4) holds for all $\pi$. In particular, because

$$
S\left(A, \pi_{k}\right)=S_{k}=D_{k}\left(A^{\prime}\right)=S\left(A^{\prime}, \delta_{k}\right),
$$

it follows that

$$
\delta_{k}=\tau \pi_{k} \sigma^{-1} \quad \text { for } k=0,1, \ldots, n-1 .
$$

Since $\delta_{0}$ is the identity permutation, this equation yields $\sigma=\tau \pi_{0}$, and therefore implies

$$
\pi_{k} \pi_{0}^{-1}=\tau^{-1} \delta_{k} \tau=\left(\tau^{-1} \delta_{1} \tau\right)^{k} \quad \text { for } k=0,1, \ldots, n-1
$$

so that (iii) holds.

For the sufficiency proof, suppose the conditions hold. Define a mapping $\tau^{-1}$ of $\{0,1, \ldots, n-1\}$ into itself by

$$
\tau^{-1}(k)=\pi_{k} \pi_{0}^{-1}(0)=\left(\pi_{1} \pi_{0}^{-1}\right)^{k}(0) .
$$

To see that $\tau^{-1}$ is a permutation (so that $\tau$ is welldefined), it suffices to observe that by their definitions $\pi_{i}$ and $\pi_{k}$, for $i \neq k$, can agree for no value of the "independent variable" (i.e., $S_{i}$ and $S_{k}$ are disjoint)

${ }^{6}$ Our convention is that the factors in a product of permutations operate in right-to-left order. If $p=0$ we take $\sigma$ to be the identity permutation; if $q=0$ we

take $\tau$ to be the identity permutation.
7 I.e., the smallest element of $S_{k+1}$ strictly exceeds the largest element of $S_{k}$, for 7 I.e., the smallest element of $S_{k+1}$ strictly exceeds the largest element of $S_{k}$, for
$k=0,1, \ldots, n-1$. This is certainly true if all entries of $A$ are distinct, but is $k=0,1, \ldots, n-1$. This is certainly true if all entries of $A$ are distinct, but is
also true (for example) if $A$ has $n$ distinct elements, each appearing in $n$ positions. and thus not at $\pi_{0}^{-1}(0)$. Now define $\sigma=\tau \pi_{0}$. Then by (iii)

$$
\tau \pi_{k} \sigma^{-1}=\tau\left(\pi_{k} \pi_{0}^{-1}\right) \tau^{-1}=\tau\left(\pi_{1} \pi_{0}^{-1}\right)^{k} \tau^{-1}
$$

so that

$$
\begin{gathered}
\tau \pi_{k} \sigma^{-1}(i)=\tau\left(\pi_{1} \pi_{0}^{-1}\right)^{k} \tau^{-1}(i)=\tau\left(\pi_{1} \pi_{0}^{-1}\right)^{k}\left(\pi_{1} \pi_{0}^{-1}\right)^{i}(0) \\
=\left(\pi_{1} \pi_{0}^{-1}\right)^{i+k}(0) \equiv i+k=\delta_{k}(i) \quad(\bmod n) .
\end{gathered}
$$

Thus eq (5) holds, and so the transform of $A$ by row permutation $\sigma$. and column permutation $\tau$ has separated and ordered diagonals.

From the first paragraph of section 2 it would seem appropriate to apply the criterion of theorem 1 to all four of the matrices $\pm A, \pm A^{T}$. Fortunately this is unnecessary; the criterion can be applied to $A$ alone without loss of information. That is, the four matrices all obey the criterion if and only if any one of them does. In checking this assertion, the equivalence of the criterion for $A$ and $\left(-A^{T}\right)$ follows by "composition" from its equivalence for $A$ and $A^{T}$, and for $A$ and $(-A)$. Since matrix negation and transposition are involutions, it suffices to prove that if $A$ obeys the three conditions of theorem 1, then the same is true of $(-A)$ and $A^{T}$. The routine but tedious verification is omitted.

We conclude this section with some examples:

$$
\text { Example 1. } A_{1}=\left(\begin{array}{rrr}
2 & 5 & 6 \\
4 & -1 & 1 \\
3 & 7 & 0
\end{array}\right) \text {. }
$$

Here $S_{0}=\{-1,0,1\}$ and $S_{2}=\{5,6,7\}$ are not chords, so that condition (ii) fails and the BKS theorem (aided by operations (i) through (iv) of the introduction) does not identify $A_{1}$ as the payoff matrix of a completely mixed game.

$$
\text { Example 2. } A_{2}=\left(\begin{array}{rrr}
4 & -3 & -2 \\
-3 & 4 & -2 \\
0 & 0 & 1
\end{array}\right) \text {. }
$$

Here condition (i) fails, for either appearance of $(-2)$ could be assigned to $S_{0}$ and the other to $S_{1}$ (see footnote 7 ); again the BKS theorem is inapplicable.

$$
\text { Example 3. } A_{3}=\left(\begin{array}{llll}
3 & 0 & 2 & 1 \\
2 & 3 & 1 & 0 \\
0 & 1 & 3 & 2 \\
1 & 2 & 0 & 3
\end{array}\right) .
$$


Here conditions (i) and (ii) are satisfied; $S_{k}$ consists of four appearances of $k$. We represent a permutation by writing each value of its "independent variable" above the corresponding value of the "dependent variable." Thus

$$
\begin{array}{lll}
\pi_{0}=\left(\begin{array}{llll}
0 & 1 & 2 & 3 \\
1 & 3 & 0 & 2
\end{array}\right) & \pi_{1}=\left(\begin{array}{llll}
0 & 1 & 2 & 3 \\
3 & 2 & 1 & 0
\end{array}\right) \\
\pi_{2}=\left(\begin{array}{llll}
0 & 1 & 2 & 3 \\
2 & 0 & 3 & 1
\end{array}\right) & \pi_{3}=\left(\begin{array}{llll}
0 & 1 & 2 & 3 \\
0 & 1 & 2 & 3
\end{array}\right) .
\end{array}
$$

We leave it to the reader to verify that all three conditions of theorem 1 are satisfied, and that using the column permutation $\tau$ defined by

$$
\tau^{-1}=\left(\begin{array}{llll}
0 & 1 & 2 & 3 \\
0 & 1 & 3 & 2
\end{array}\right)
$$

and the row permutation

$$
\sigma=\tau \pi_{0}=\left(\begin{array}{llll}
0 & 1 & 2 & 3 \\
1 & 2 & 0 & 3
\end{array}\right),
$$

as in the sufficiency proof of theorem 1, leads to

$$
A_{3}^{\prime}=\left(\begin{array}{llll}
0 & 1 & 2 & 3 \\
3 & 0 & 1 & 2 \\
2 & 3 & 0 & 1 \\
1 & 2 & 3 & 0
\end{array}\right),
$$

a transform of $A$ with separated and ordered diagonals.

\section{Extensions of the BKS theorem}

The previous section was essentially combinatorial; the present one, in contrast, has some specifically game-theoretic content. We begin with the following simple generalization of the BKS theorem: ${ }^{8}$

Theorem 2: The game with n-by-n matrix $\mathrm{A}$ is completely mixed if there exist integers $\mathrm{p}, \mathrm{q}, \mathrm{r}, \mathrm{s}$ with sum relatively prime to $\mathrm{n}$, such that

(a) $\mathrm{a}_{\mathrm{ij}}<\mathrm{a}_{\mathrm{i}, \mathrm{j}+\mathrm{p}}$ whenever $\mathrm{j}-\mathrm{i} \not \equiv \mathrm{q}(\bmod \mathrm{n})$,

(b) $\mathrm{a}_{\mathrm{ij}}>\mathrm{a}_{\mathrm{i}+\mathrm{r}, \mathrm{j}}$ whenever $\mathrm{i}-\mathrm{j} \neq \mathrm{s}(\bmod \mathrm{n})$.

Before proving this result, we observe that it implies the BKS theorem; if $A$ has separated and ordered diagonals, then theorem 2 applies with $p=r=1, q=n-1$, and $s=0$. Theorem 2 is strictly stronger than the BKS theorem, for it applies (also with $p=r=1, q=n-1$, and $s=0)$ to show that the matrix $A_{1}$ of example 1 represents a completely

\footnotetext{
${ }^{8}$ In what follows, appropriate numbers should be read modulo $n$.
}

mixed game, a fact which the BKS theorem (even aided by row and column, permutations, matrix transposition, and negation) could not reveal, as was demonstrated in section 2.

For the proof, suppose that some component $x_{k}^{*}$ of an optimal strategy $X^{*}$ vanished. We will show that this implies

$$
x_{k+p+q+r+s}^{*}=0 .
$$

The same argument can then be applied with $k+p+q+r+s$ replacing $k$. Repetition of the argument, together with the hypothesis on $p+q+r+s$, shows that all components of $X^{*}$ vanish, which is absurd since these components must sum to unity. Thus no component of any optimal $X^{*}$ can vanish; a similar analysis applies for optimal $Y^{*}$, and so the game is completely mixed.

To prove eq (7), first use (1) and the optimality of $X^{*}$ to write

$$
v \leq \sum_{i=0}^{n-1} x_{i}^{*} a_{i, k+q}=\sum_{i \neq k} x_{i}^{*} a_{i, k+q} .
$$

By hypothesis (a) of the theorem we have $a_{i, k+q}$ $<a_{i, k+p+q}$ for each term in the last sum, and at least one of the $x_{i}^{*}$ in this sum is positive (they are all nonnegative). Therefore

$$
v<\sum_{i \neq k} x_{i}^{*} a_{i, k+p+q}=\sum_{i=0}^{n-1} x_{i}^{*} a_{i, k+p+q} .
$$

By the remark immediately following (1) and (2), $y_{k+p+q}^{*}$ vanishes for every optimal $Y^{*}$, so that by (2)

$$
v \geq \sum_{j=0}^{n-1} a_{k+p+q+s, j} y_{j}^{*}=\sum_{\neq j k+p+j q} a_{k+p+q+s, j} y_{j}^{*} .
$$

By hypothesis (b) of the theorem we have $a_{k+p+q+s, j}>a_{k+p+q+r+s, j}$ for each term in the last sum, and at least one of the $y_{j}^{*}$ in this sum is positive (they are all nonnegative). Therefore

$$
v>\sum_{j \neq k+p+q} a_{k+p+q+r+s, j} y_{j}^{*}=\sum_{j=0}^{n-1} a_{k+p+q+r+s, j} y_{j}^{*},
$$

which inplies eq (7) by the comment following (1) and (2). This completes the proof of the theorem.

To derive full benefit from theorem 2 , we should determine its range of applicability when aided by the four operations listed in the introduction. These aims are accomplished in the next theorem, which is related to theorem 2 as theorem 1 is related to the BKS theorem. First two lemmas will be given.

Lemma 1. Suppose that each row and each column of $\mathrm{A}$ has a unique minimum entry, that the row minima form a chord, and that the column minima form a chord. Then the row and column minima coincide.

For the proof, suppose for example that $a_{i(0), j(0)}$ is a row minimum but not a column minimum. Let $a_{i(1), j(0)}$, with $i(1) \neq i(0)$, be the minimum of column $j(0)$. It is not the minimum of row $i(1)$, since column $j(0)$ contains only one row minimum (the one in row $i(0))$. Let $a_{i(1), j(1)}$, with $j(1) \neq j(0)$, be 
the minimum of row $i(1)$. It is not the minimum of column $j(1)$, etc. Continuing similarly, we obtain an infinite sequence of entries of $A$, which is strictly decreasing and therefore nonrepeating. Since this is impossible, every row minimum $a_{i(0), j(0)}$ must also be a column minimum.

Lemma 2. Suppose that each line of A has distinct entries, so that the sets $\mathbf{R}_{\mathrm{k}}$ of $\mathrm{k}$-th smallest row entries ${ }^{10}$ and $\mathrm{C}_{\mathrm{k}}$ of $\mathrm{k}$-th smallest column entries are uniquely defined for $0 \leq \mathrm{k}<\mathrm{n}$. If each $\mathrm{R}_{\mathrm{k}}$ and each $\mathrm{C}_{\mathrm{k}}$ is a chord, then $\mathrm{R}_{\mathrm{k}}=\mathrm{C}_{\mathrm{k}}$ for all $\mathrm{k}$.

For, $R_{0}=C_{0}$ by lemma 1 . Now replace all entries in $R_{0}=C_{0}$ by numbers greater than any other entries of $A$. The result is a matrix $A^{*}$ for which (with an obvious notation) $R_{k-1}\left(A^{*}\right)=R_{k}(A)$ and $C_{k-1}\left(A^{*}\right)=C_{k}(A)$ for $1 \leq k \leq n-1$, while $R_{n-1}\left(A^{*}\right)=$ $C_{n-1}\left(A^{*}\right)$ consists of the new entries. By lemma 1,

$$
R_{1}(A)=R_{0}\left(A^{*}\right)=C_{0}\left(A^{*}\right)=C_{1}(A),
$$

and the argument can be repeated until the proof is complete.

Theorem 3: Some transform of A obeys the conditions of theorem 2, if and only if

(i) the sets $\mathrm{R}_{\mathrm{k}}$ and $\mathrm{C}_{\mathrm{k}}$ are well-defined for $0 \leq \mathrm{k}<\mathrm{n}$,

(ii) each $\mathrm{R}_{\mathrm{k}}$ and each $\mathrm{C}_{\mathrm{k}}$ is a chord, so that a unique permutation $\rho_{\mathrm{k}}$ is defined by $\mathrm{R}_{\mathrm{k}}=\mathrm{S}\left(\mathrm{A}, \rho_{\mathrm{k}}\right)$, and

(iii) $\rho_{\mathrm{k}} \rho_{0}^{-1}=\left(\rho_{1} \rho_{0}{ }^{-1}\right)^{k}$ for $0 \leq \mathrm{k}<\mathrm{n}$.

If all the $\pi_{k}$ of theorem 1 exist, then clearly $\pi_{k}=\rho_{k}$. Thus the real distinction between theorems 1 and 3 lies in conditions (i) and (ii), and the illustration just after the statement of theorem 2 was a "typical" one.

The necessity of the three conditions will be proved first. ${ }^{11}$ Suppose transform $A^{\prime}=\left(a_{i j}^{\prime}\right)$ of $A$ obeys (a) and (b) of theorem 2. It will be shown initially that this implies, for each $i$ and $j$,

$$
\begin{aligned}
& a_{i,{ }_{i+q}}^{\prime}>a_{i, i+q-p}^{\prime}>\ldots>a_{i,{ }_{i+q-(n-1) p}^{\prime},}>a_{j+s-(n-1) r, j}^{\prime} ; \\
& a_{j+s, j}^{\prime}<a_{j+s-r, j}^{\prime}<\ldots<a_{j}^{\prime}
\end{aligned}
$$

only (8) need be discussed in detail. By (a) it follows that only $a_{i, i+p}^{\prime}$ can be a largest entry of the $i$ th row of $A^{\prime}$. If $p$ is relatively prime to $n$, then (8) follows readily from (a). If on the other hand there is a least residue $k$, with $1 \leq k<n$, such that $n$ divides $k p$, then a contradiction is obtained ${ }^{12}$ by choosing $j$ different from $i+q-\nu p$ for $0 \leq \nu<k$ and using (a) of theorem 2 to write

$$
a_{i j}^{\prime}<a_{i, j+p}^{\prime}<\ldots<a_{i, j+k p}^{\prime}=a_{i j}^{\prime} .
$$

Thus (8) is proved.

By (8) and (9), each row and column of $A^{\prime}$ (and thus of $A$ ) has all its entries distinct; condition (i) has been verified. By the nature of row and column permutations, $A$ will satisfy condition (ii) if $A^{\prime}$ does.

\footnotetext{
${ }^{9}$ A line of a matrix is either a row or a column.

${ }_{10} R_{0}$ consists of the smallest entry of the top row of $A$, the smallest entry of the next row, etc. $R_{1}$ consists of the next-to-smallest entry of the top row, the next-to-smallest entry of the next row etc.

11. In the necessity proof the fact that $p+q+r+s$ is relatively prime to $n$ is not In the to $n$ is not used. This fact is actually a consequence of conditions (a) and (b) of theorer
2 , which also (see eq (10)) imply that $p=r$ and that $p+q+s$ is divisible by $n$.
}

2 , which also (see eq (10)) imply that $p=r$ an
12 The assumption $n>1$ is tacit throughout.
Now the $k$ th smallest entry in the $i$ th row of $A^{\prime}$ is $a_{i, i+q-(n-1-k) p}^{\prime}$; if some $R_{k}$ were $n o t$ a chord we would have

$$
i+q-(n-1-k) p \equiv t+q-(n-1-k) p \quad(\bmod n)
$$

for distinct residues $i$ and $t$, which is impossible. Similarly each $C_{k}$ is a chord. Thus only the necessity of (iii) remains to be justified.

There exist permutations $\sigma$ and $\tau$ such that eq (4) holds for all permutations $\pi$. In particular, by lemma 2 ,

$$
\begin{aligned}
& R_{k}=S\left(A, \rho_{k}\right)=S\left(A^{\prime}, \tau \rho_{k} \sigma^{-1}\right), \\
& C_{k}=S\left(A, \rho_{k}\right)=S\left(A^{\prime}, \tau \rho_{k} \sigma^{-1}\right) .
\end{aligned}
$$

Displays (8) and (9) show that

$$
\begin{aligned}
& R_{k}=S\left(A^{\prime}, \delta_{q}\left(\delta_{(n-k-1) p}\right)^{-1}\right)=S\left(A^{\prime}, \delta_{q}\left(\delta_{p}\right)^{k+1}\right), \\
& C_{k}=S\left(A^{\prime},\left(\delta_{s}\right)^{-1} \delta_{k r}\right)=S\left(A^{\prime},\left(\delta_{s}\right)^{-1}\left(\delta_{r}\right)^{k}\right),
\end{aligned}
$$

so that we have

$$
\begin{aligned}
\tau \rho_{k} \sigma^{-1}=\delta_{q}\left(\delta_{p}\right)^{k+1}, \tau \rho_{k} \sigma^{-1}=\left(\delta_{s}\right)^{-1}\left(\delta_{r}\right)^{k} & \\
& \text { for } k=0,1, \ldots, n-1 .
\end{aligned}
$$

Setting $k=0$ in the first of these equations yields $\sigma=\left(\delta_{p+q}\right)^{-1} \tau \rho_{0}$, so that (10) yields

$$
\rho_{k} \rho_{0}^{-1}=\left(\tau^{-1} \delta_{p} \tau\right)^{k}
$$

and condition (iii) is satisfied.

For the sufficiency proof, suppose the three conditions hold. As in the proof of theorem 1, we define a mapping $\tau^{-1}$ of $\{0,1, \ldots, n-1\}$ into itself by

$$
\tau^{-1}(k)=\rho_{k} \rho_{0}^{-1}(0)=\left(\rho_{1} \rho_{0}^{-1}\right)^{k}(0),
$$

and observe that $\tau^{-1}$ is a permutation so that $\tau$ is well-defined. We have

$$
\delta_{k}(i)=\tau\left(\rho_{1} \rho_{0}^{-1}\right)^{k+i}(0)=\tau\left(\rho_{k} \rho_{0}^{-1}\right) \tau^{-1}(i)
$$

so that $\delta_{k}=\tau\left(\rho_{k} \rho_{0}^{-1}\right) \tau^{-1}$; equivalently $\rho_{k} \rho_{0}{ }^{-1}=\tau^{-1} \delta_{k} \tau$. On defining $\sigma=\left(\delta_{1}\right)^{-1} \tau \rho_{0}$, we find that the first of eqs (10) holds with $p=1$ and $q=0$. By setting $r=1$ and $s=n-1$ we also satisfy the second of eq (10), so that conditions (a) and (b) of theorem 2 hold for the transform of $A$ under row permutation $\sigma$ and column permutation $\tau$. Since

$$
p+q+r+s=n+1
$$

is relatively prime to $n$, the proof is complete.

Two comments are in order. First, the last paragraph shows that some transform of $A$ obeys theorem 2 for some $(p, q, r, s)$ if and only if at least one transform does so with $p=r=1, q=0, \quad s=n-1$. Second, there is no need to apply the criterion of theorem 3 to $(-A)$ and $\pm A^{T}$ as well as $A$; the situation is just like the one mentioned directly after the proof of theorem 1 . 


\section{Related Results}

The proof of theorem 2 will obviously remain valid if $p, q, r$, and $s$ are permitted to vary with $i$ and $j$ in appropriate ways. This leads to the sufficient condition for complete mixture contained in the following theorem.

Theorem 4: Let $\mathrm{p}, \mathrm{q}, \mathrm{r}$ and $\mathrm{s}$ be permutations of $\{0,1, \ldots, \mathrm{n}-1\}$ and consider a game with n-by-n matrix A such that
(a) $\mathrm{a}_{\mathrm{ij}}<\mathrm{a}_{\mathrm{i}, \mathrm{p}(\mathrm{j})}$
whenever $\mathrm{j} \neq \mathrm{q}(\mathrm{i})$,
(b) $\mathrm{a}_{\mathrm{ij}}<\mathrm{a}_{\mathrm{r}(i), j}$
whenever $\mathrm{i} \neq \mathrm{s}(\mathrm{j})$.

If rspq is a cyclic permutation then no optimal $\mathrm{X}^{*}$ has a zero component, while if pqrs is cyclic then no optimal $\mathrm{Y}^{*}$ has a zero component.

The proof is so like that of theorem 2 that it can be omitted; $q$ and $s$ must be permutations (and not merely mappings of $\{0,1 \ldots, n-1\}$ into itself) to permit the same to be true of $r s p q$ or pqrs.

One would expect the criterion of theorem 4 (aided by the four operations listed in the introduction) to be more powerful than that of theorem 2 (similarly aided). In particular, it would seem possible that some transform of $A$ (and thus $A$ itself) could be proved to have no zero component in any optimal $X^{*}$ by means of this criterion, and some other transform of $A$ (and thus $A$ itself) proved to have no zero component in any optimal $Y^{*}$. All these hopes are dashed by the following result.

Theorem 5: Some transform of $\mathbf{A}$ obeys $(a)$ and $(b)$ of theorem 4 for some p,q,r,s if and only if some transform of $\mathrm{A}$ obeys the condition of theorem 2.

The correctness in the "if" direction is trivial, since theorem 2 is the special case of theorem 4 in which the relevant permutations are diagonal. So we need only assume that some transform $A^{\prime}=$ $\left(a_{i j}^{\prime}\right)$ of $A$ obeys (a) and (b) of theorem 4, and deduce that $A$ obeys the three conditions of theorem 3 .

Condition (a) implies that only $q(i)$ can be left fixed by $p$; since $q(0) \neq q(1)$, no symbol is left fixed by $p$. Therefore, since by (a) no element of the $i$ th row of $A^{\prime}$ except $a_{i . q(i)}^{\prime}$ can be the row's largest entry, we see that the sequence of subscripts

$$
(i, i+q), \quad(i, i+q-p), \ldots(i, i+q-(n-1) p)
$$

in (8) now becomes ${ }^{13}$

$$
(i, q(i)), \quad\left(i, p^{-1} q(i)\right), \ldots\left(i, p^{-(n-1)} q(i)\right) .
$$

If $\sigma$ and $\tau$ are the row and column permutations leading from $A$ to $A^{\prime}$, then we find that all $\rho_{k}$ are well-defined with

$$
\tau \rho_{k} \sigma^{-1}=p^{k+1-n} q \quad \text { for } k=0,1, \ldots, n-1
$$

${ }^{13}$ The detailed argument used earlier to justify (8) is easily generalized to support (11). in analogy with the first part of (10). From this it follows that condition (iii) of theorem 3 is obeyed.

Similarly, using (b) and the appropriate generalization of (9), we find that the $C_{k}$ 's are well-defined chords, so that (i) and (ii) of theorem 3 holds and the proof is complete.

Bohnenblust, Karlin, and Shapley (op. cit., footnote 4) give a second sufficient condition for complete mixture, that of "main diagonal separated and dominant." To state it precisely, let $\mu=\mu(A)$ denote the largest of the entries of $A$ off the main diagonal; then the condition consists of

$$
a_{i i}>\mu \quad \text { for } i=0,1, \ldots, n-1
$$

and either of the situations

$$
\begin{array}{ll}
\sum_{i=0}^{n-1} a_{i j}>n \mu & \text { for } j=0,1, \ldots, n-1, \\
\sum_{j=0}^{n-1} a_{i j}>n \mu & \text { for } i=0,1, \ldots, n-1 .
\end{array}
$$

It is interesting to note that the matrix $A_{1}$ of example 1 , which defied the BKS condition extended by theorem 1 but which yielded to our theorem 2, would also prove intractible to this condition. For $S_{0}$ and $S_{2}$ are not chords of $A_{1}$ (see the discussion of example 1), whereas it is easily shown that $S_{0}$ or $S_{n-1}$ must be a chord if some transform of any of $n$-by- $n \pm A, \pm A^{T}$ is to obey (12).

It is natural to ask whether theorems 2 and 3 are sufficiently stronger than the BKS theorem to achieve identification of all completely mixed 3-by-3 games. This is unfortunately not the case; the completely mixed game whose payoff matrix is the $A_{2}$ of example 2, cited as a "maverick" by Bohnenblust, Karlin, and Shapley (op. cit.), is not tamed by our results. To see this, note that $A_{2}$ (and therefore $-A_{2}$ and $\pm A_{2}^{T}$ as well) fails to satisfy condition (i) of theorem 3 .

A slight twist in the proof of theorem 2 leads to sharper analogs of theorems 2 and 4; for brevity, only the latter will be given (the former is obtained by specializing the relevant permutations to be diagonal).

Theorem 6: Let $\mathrm{p}, \mathrm{q}, \mathrm{r}$ and $\mathrm{s}$ be permutations of $\{0,1, \ldots, n-1\}$ and consider a game with $\mathrm{n}-\mathrm{b} y-\mathrm{n}$ matrix A such that

$$
\begin{aligned}
& \text { (a) } \mathrm{a}_{\mathrm{ij}} \leq \mathrm{a}_{\mathrm{i}, \mathrm{p}(\mathrm{j})} \quad \text { whenever } \mathrm{j} \neq \mathrm{q}(\mathrm{i}) \text {, } \\
& \text { (a') } \mathrm{a}_{\mathrm{rsp}(\mathrm{j}), \mathrm{j}}<\mathrm{a}_{\mathrm{rsp}(\mathrm{j}), \mathrm{p}(\mathrm{j})} \quad \text { for all } \mathrm{j}, \\
& \text { (b) } \mathrm{a}_{\mathrm{ij}}>\mathrm{a}_{\mathrm{r}(\mathrm{1}), \mathrm{j}} \quad \text { whenever } \mathrm{i} \neq \mathrm{s}(\mathrm{j})
\end{aligned}
$$

If rspq is a cyclic permutation, then no optimal $\mathrm{X}^{*}$ has a zero componert.

Theorem 7: Let $\mathrm{p}, \mathrm{q}, \mathrm{r}$ and $\mathrm{s}$ be permutations of $\{0,1, \ldots, \mathrm{n}-1\}$ and consider a game with $\mathrm{n}-b y-\mathrm{n}$ matrix A such that 

(a) $\mathrm{a}_{1 \mathrm{j}}<\mathrm{a}_{\mathrm{i}, \mathrm{p}(\mathrm{j})} \quad$ whenever $\mathrm{j} \neq \mathrm{q}(\mathrm{i})$,
(b) $\mathrm{a}_{\mathrm{ij}} \geq \mathrm{a}_{\mathrm{r}(\mathrm{l}), \mathrm{j}} \quad$ whenever $\mathrm{i} \neq \mathrm{s}(\mathrm{j})$,
( $\left.\mathrm{b}^{\prime}\right) \quad \mathrm{a}_{1, \operatorname{pqr}(\mathbf{1})}>\mathrm{a}_{\mathrm{r}(\mathbf{1}), \operatorname{pqr}(\mathrm{i})} \quad$ for all $\mathrm{i}$.

If pqrs is a cyclic permutation, then no optimal $\mathrm{Y}^{*}$ has a zero component.

Only theorem 6 need be proved. In view of the hypothesis on $r s p q$, it suffices to prove that if $x_{k}^{*}=0$ for some optimal $X^{*}$, then $x_{r s p q(k)}^{*}=0$ as well. By (1) and the optimality of $X^{*}$, we have

$$
v \leq \sum_{i=0}^{n-1} x_{i}^{\star} a_{i, q(k)}=\sum_{i \neq k} x_{i}^{*} a_{i, q(k)} .
$$

In the right hand expression, all $x_{i}^{*}$ are nonnegative and we have $a_{i, q(k)} \leq a_{i, p q(k)}$ for each term, with strict inequality for $i=r s p q(k)$ (which is present since $r s p q$ does not leave $k$ fixed). Thus, unless $x_{r s p q(k)}^{*}=0$ (i.e., if $x_{r s p q(k)}^{*}>0$ ), we have

$$
v<\sum_{i \neq k} x_{i}^{*} a_{i, p q(k)}=\sum_{i=0}^{n-1} x_{i}^{*} a_{i, p q(k)},
$$

and the rest of the proof is as for theorem 2, leading to the conclusion $x_{r s p q(k)}^{*}=0$.

Condition (b) of theorem 6 implies that each column of $A$ has distinct entries, while condition (a) of theorem 7 implies that each row has distinct entries. Thus the matrix $A_{2}$ eludes these theorems (even when aided by the four operations). It remains unclear whether all 3-by-3 completely mixed games can be characterized along the lines pursued above.
Addendum. I am indebted to $\mathrm{K}$. Goldberg (NBS Numerical Analysis Section) for the following observations.

If $A$ is nonsingular, $v \neq 0$, and all row and column sums of $A^{-1}$ are nonzero and have the same sign, then the game is completely mixed. ${ }^{14}$ This covers examples $A_{1}$ and $A_{2}$ of section 2 ; it is not an "inspection" method since a matrix inversion is required. For $n=3$, however, an "inspective" formulation can be given; if

$$
A=\left(\begin{array}{lll}
a_{1} & b_{1} & c_{1} \\
c_{2} & a_{2} & b_{2} \\
b_{3} & c_{3} & a_{3}
\end{array}\right)
$$

then what is required is that all $2-b y-2$ principal minors of

$\left(\begin{array}{lll}b_{1}-c_{1} & c_{1}-a_{1} & a_{1}-b_{1} \\ a_{2}-b_{2} & b_{2}-c_{2} & c_{2}-a_{2} \\ c_{3}-a_{3} & a_{3}-b_{3} & b_{3}-c_{3}\end{array}\right)$ and $\left(\begin{array}{lll}c_{2}-b_{3} & b_{3}-a_{1} & a_{1}-c_{2} \\ a_{2}-c_{3} & c_{3}-b_{1} & b_{1}-a_{2} \\ b_{2}-a_{3} & a_{3}-c_{1} & c_{1}-b_{2}\end{array}\right)$

have the same (strict) sign.

14 K. Goldberg, Random notes on matrices, J. Research NBS 60 (1958) R P2850. Theorem 2 of that paper also can apply to the individual players.

(Paper 67B1-90) 